\title{
Article \\ MicroRNA Profiles of Maternal and Neonatal Endothelial Progenitor Cells in Preeclampsia
}

\author{
Lars Brodowski ${ }^{1,2, \dagger}$, Bianca Schröder-Heurich ${ }^{1,+}{ }^{\oplus}$, Sandra von Hardenberg ${ }^{1}$, Katja Richter ${ }^{1}$, \\ Constantin S. von Kaisenberg ${ }^{2}$, Oliver Dittrich-Breiholz ${ }^{3}$, Nadia Meyer ${ }^{1}$, Thilo Dörk ${ }^{1}$ (D) \\ and Frauke von Versen-Höynck 1,2,*(D)
}

check for

updates

Citation: Brodowski, L.;

Schröder-Heurich, B.; von

Hardenberg, S.; Richter, K.; von

Kaisenberg, C.S.; Dittrich-Breiholz, O.;

Meyer, N.; Dörk, T.; von

Versen-Höynck, F. MicroRNA Profiles of Maternal and Neonatal Endothelial Progenitor Cells in Preeclampsia. Int. J. Mol. Sci. 2021, 22, 5320. https:// doi.org/10.3390/ijms22105320

Academic Editor: Jolanta Weaver

Received: 20 March 2021

Accepted: 12 May 2021

Published: 18 May 2021

Publisher's Note: MDPI stays neutral with regard to jurisdictional claims in published maps and institutional affiliations.

Copyright: (C) 2021 by the authors Licensee MDPI, Basel, Switzerland. This article is an open access article distributed under the terms and conditions of the Creative Commons Attribution (CC BY) license (https:// creativecommons.org/licenses/by/ $4.0 /)$
1 Gynecology Research Unit, Hannover Medical School, Carl-Neuberg-Strasse 1, D-30625 Hannover, Germany; Brodowski.Lars@mh-hannover.de (L.B.); Schroeder-Heurich.Bianca@mh-hannover.de (B.S.-H.); vonHardenberg.Sandra@mh-hannover.de (S.v.H.); Richter.Katja@mh-hannover.de (K.R.); Meyer.Nadia@mh-hannover.de (N.M.); Doerk.Thilo@mh-hannover.de (T.D.)

2 Department of Obstetrics and Gynecology, Hannover Medical School, Carl-Neuberg-Strasse 1, D-30625 Hannover, Germany; vonKaisenberg.Constantin@mh-hannover.de

3 Research Core Unit Genomics, Hannover Medical School, Carl-Neuberg-Strasse 1, D-30625 Hannover, Germany; Dittrich.Oliver@mh-hannover.de

* Correspondence: vonVersen-Hoeynck.Frauke@mh-hannover.de; Tel.: +49-511-532-8703; Fax: +49-511-532-6081

+ These authors contribute equally.

Abstract: Preeclampsia is associated with an increased cardiovascular morbidity of mother and offspring, thus contributing to a substantial burden in women and children's health. It has been proven that endothelial progenitor cell (EPC) numbers and functional characteristics are impaired in cardiovascular disease and preeclampsia, although causative factors for the latter have remained elusive. MicroRNA (miRNA) modifications are a potential mechanism through which exposure to an altered environment translates into the development of chronic disease. In this study, we examined whether development of preeclampsia corresponds to alterations of miRNAs in maternaland cord-blood-derived EPC. To test this end, we analyzed maternal and neonatal miRNAs via RNA sequencing from endothelial cells of preeclamptic and healthy controls in different cell culture passages. We were able to demonstrate differentially represented miRNAs in all groups. Hsa-miR1270 showed significantly different levels in cord blood EPC from preeclampsia versus control and was negatively correlated with mRNA levels of its predicted targets ANGPTL7 and TFRC. Transfection with an hsa-miR-1270 inhibitor decreased the tube formation capacity and chemotactic motility but did not change proliferation in vitro. Target predictions and gene set enrichment analyses identified alternative splicing as a significantly enriched pathway for hsa-miR-1270. The top miRNAs in three other groups were predicted to target transcriptional and developmental pathways. Here, we showed for the first time significantly different levels of miRNAs and differently represented mRNA levels of predicted target genes in EPC derived from preeclampsia. Understanding the effects of preeclampsia on the epigenetic mechanisms of EPC will be crucial and may provide initial insights for further evaluation of the benefits of therapies targeting this cell population.

Keywords: preeclampsia; cardiovascular morbidity; endothelial progenitor cells; miRNA; hsa-miR1270

\section{Introduction}

A woman's obstetrical history is an important part of her and her offspring's risk profile for future cardiovascular disease. Preeclampsia is a severe pregnancy-specific disorder arising in the latter half of pregnancy, manifested by new-onset hypertension with proteinuria or other organ manifestations. Preeclampsia is the second leading cause of maternal and fetal morbidity and mortality in pregnancy and has long-term, adverse health implications for both mother and offspring [1,2]. Recent studies indicate that 
preeclampsia is not only an independent cardiovascular risk factor for the mother, but also contributes to an increased cardiovascular risk for the offspring [2-4]. Substantial evidence supports endothelial cell dysfunction as being a central feature of the pathogenesis of preeclampsia [5], as well as a risk factor for adverse cardiovascular events [5-7].

Endothelial progenitor cells (EPC) are essential to maintain a healthy endothelium over an individual's lifetime. EPC have been extensively studied for almost 20 years and are considered a potential marker for endothelial regeneration ability [8]. Both a decreased number and impaired function of circulating EPC have been reported in patients with cardiovascular disease [9]. The level of circulating EPC predicts the occurrence of cardiovascular events and death from cardiovascular causes and may help to identify patients at increased cardiovascular risk $[6,7,10]$. Decreased cell numbers and colony-forming units of maternal EPC are described as a sign of impaired endothelial repair capacity in preeclampsia $[10,11]$. Our own research shows that endothelial colony-forming cells (ECFC), a proliferative EPC subtype, are reduced in cord blood of infants born to women with preeclampsia [12]. Further, we described key functional differences and differential DNA methylation profiles between ECFC obtained from cord blood of preeclamptic compared to healthy pregnancies [12,13].

Micro RNAs (miRNAs) are evolutionarily conserved, noncoding RNA molecules ( 22 nucleotides long, single-stranded) that regulate gene expression through base pairing with complementary sequences in their target mRNA, leading to translational repression or transcript degradation [14]. MiRNA analyses indicate that a variety of pathological tissues display miRNA expression profiles that are significantly different from normal tissues and cells [15], which may be useful for a wide range of applications in clinical diagnostics and therapy $[16,17]$. Furthermore, emerging evidence has uncovered miRNAs as new targets or regulators of cardiovascular medications, given the ability of miRNAs to interact with certain cardiovascular drugs $[18,19]$. Several reports have also proposed a role for miRNAs in pregnancy complications such as preeclampsia.

In order to capitalize on the therapeutic potential of targeting ECFC in preeclampsia, the main objective of this study was to determine the global miRNA profile of maternalblood- and cord-blood-derived ECFC for preeclampsia and healthy pregnancies (control) using small RNA sequencing. Furthermore, we compared miRNA profiles between maternal and cord blood ECFC and examined whether cell culture passage $(\mathrm{P})$ affects miRNA patterns to gain important information for future use in cell-based therapies. We additionally assessed whether known target genes of differently represented miRNAs are affected. Subsequently, control ECFC were transfected with an inhibitor of the miRNA that was most highly abased in preeclampsia versus (vs.) control and ECFC function was evaluated by tube formation and cell proliferation assays in vitro. Lastly, we explored associations between altered miRNAs and pathways affected in cardiovascular disease.

\section{Results}

\subsection{Demographic Characteristics}

Women with preeclampsia $(n=12)$ and women with healthy, uncomplicated pregnancies $(n=9)$ were enrolled for this study. Depending on the availability of maternaland cord-blood-derived ECFC, six pregnancies were evaluated further. Demographic and clinical characteristics are summarized in Table 1. Gestational age was significantly lower in preeclampsia in both the cord blood group $(p=0.03)$ and in the group of maternal-bloodderived ECFC $(p=0.01)$. Birth weight was lower $(p=0.004)$ in the group of maternalblood-derived ECFC in pregnancies complicated by preeclampsia. Diastolic $(p=0.001)$ and systolic blood pressures $(p=0.001)$ were significantly higher in preeclampsia group. 
Table 1. Patient demographics of cord-blood- and maternal-blood-derived ECFC. Data are expressed as means $+/-$ standard deviation or numbers $(n)$ and \%. BMI, body mass index; ECFC, endothelial progenitor cells; SBP, systolic blood pressure; DBP, diastolic blood pressure.

\begin{tabular}{|c|c|c|c|c|c|c|}
\hline & \multicolumn{3}{|c|}{ Cord-Blood-Derived ECFC } & \multicolumn{3}{|c|}{ Maternal-Blood-Derived ECFC } \\
\hline & $\begin{array}{c}\text { Healthy } \\
\text { Pregnancy } \\
(n=6)\end{array}$ & $\begin{array}{l}\text { Preeclamptic } \\
\text { Pregnancy } \\
(n=6)\end{array}$ & $p$-Value & $\begin{array}{c}\text { Healthy } \\
\text { Pregnancy } \\
(n=6)\end{array}$ & $\begin{array}{l}\text { Preeclamptic } \\
\text { Pregnancy } \\
(n=6)\end{array}$ & $p$-Value \\
\hline Maternal age at delivery (years) & $32.8 \pm 5.2$ & $31.5 \pm 3.7$ & 0.65 & $31.7 \pm 7.4$ & $30.8 \pm 5.5$ & 0.85 \\
\hline Gestational age at delivery (weeks) & $38.6 \pm 0.5$ & $36.8 \pm 1.1$ & 0.03 & $38.3 \pm 0.7$ & $33.1 \pm 3.9$ & 0.01 \\
\hline Multiparous $n(\%)$ & $5(83 \%)$ & $4(67 \%)$ & 1.00 & $6(100 \%)$ & $4(67 \%)$ & 0.45 \\
\hline Maternal pre-pregnancy BMI $\left(\mathrm{kg} / \mathrm{m}^{2}\right)$ & $26.2 \pm 6.3$ & $29.7 \pm 12.9$ & 0.80 & $27.2 \pm 4.5$ & $26.9 \pm 6.1$ & 0.90 \\
\hline Gestational SBP, pre-delivery $(\mathrm{mmHg})$ & $121 \pm 8$ & $164 \pm 18$ & $<0.001$ & $121 \pm 8$ & $163 \pm 11$ & $<0.001$ \\
\hline $\begin{array}{l}\text { Gestational SBP, before } 20 \text { week } \\
\text { gestation }(\mathrm{mmHg})\end{array}$ & $112 \pm 11$ & $125 \pm 21$ & 0.20 & $116 \pm 14$ & $113 \pm 16$ & 0.70 \\
\hline $\begin{array}{l}\text { Gestational DBP, pre-delivery } \\
(\mathrm{mmHg})\end{array}$ & $70 \pm 5$ & $98 \pm 6$ & $<0.001$ & $74 \pm 9$ & $95 \pm 13$ & 0.009 \\
\hline $\begin{array}{c}\text { Gestational DBP, before } 20 \text { week } \\
\text { gestation }(\mathrm{mmHg})\end{array}$ & $69 \pm 11$ & $80 \pm 10$ & 0.10 & $68 \pm 11$ & $76 \pm 9$ & 0.18 \\
\hline Birth weight (g) & $3487 \pm 523$ & $2741 \pm 669$ & 0.06 & $3314 \pm 651$ & $1628 \pm 815$ & 0.004 \\
\hline Birth weight percentile & $55 \pm 30$ & $35 \pm 35$ & 0.32 & $51 \pm 35$ & $21 \pm 16$ & 0.11 \\
\hline Birth weight percentile $<10$ th $n(\%)$ & $0(0 \%)$ & $1(17 \%)$ & 1.00 & $1(17 \%)$ & $2(34 \%)$ & 1.00 \\
\hline Caesarean delivery $n(\%)$ & $6(100 \%)$ & $5(83 \%)$ & 1.00 & $6(100 \%)$ & $6(100 \%)$ & 1.00 \\
\hline Maternal race, White $n(\%)$ & $6(100 \%)$ & $5(83 \%)$ & 1.00 & $6(100 \%)$ & $6(100 \%)$ & 1.00 \\
\hline Baby sex, Male $n(\%)$ & $3(50 \%)$ & $1(17 \%)$ & 0.55 & $1(17 \%)$ & $2(34 \%)$ & 1.00 \\
\hline
\end{tabular}

\subsection{ECFC Isolation and Characterization}

ECFC emerged in culture as discrete, late-outgrowth colonies displaying the characteristic cobblestone morphology. Flow cytometric results showed that ECFC in P3 and P5 were homogenous and had the typical phenotype of endothelial cells, namely CD31+, CD45-, and CD133- (Supplemental Figure S1A). The number of days until first appearance of colonies ( 50 characteristic cells) of cord blood ECFC was significantly lower in preeclampsia, while in maternal blood ECFC colonies trended to appear later in preeclampsia compared with controls (cord blood ECFC: $7.6 \pm 1.6$ (control) vs. $10.8 \pm 2.6$ (preeclampsia); $p=0.04$; maternal blood ECFC: $13.2 \pm 4.3$ (control) vs. $15.2 \pm 1.7$ (preeclampsia); $p=0.31$ ). There were no differences in the total numbers of cord blood and maternal blood ECFC colonies (cord blood ECFC: $8.2 \pm 2.3$ (control) vs. $10.2 \pm 6.8$ (preeclampsia); $p=0.5$; maternal blood ECFC: $1.5 \pm 0.8$ (control) vs. $1.8 \pm 1.3$ (preeclampsia); $p=0.69$ ) (Supplemental Figure S1B).

\subsection{MiRNA Profiles in the Comparison Groups}

Our study had three objectives. We first determined whether pregnancies complicated by preeclampsia have different miRNA profiles compared to healthy pregnancies. Furthermore, we analyzed if there are differences in the miRNA profiles between maternal and cord-blood-derived ECFC, as well as between P3 and P5. Venn diagrams depict the number of common and different miRNA expression changes in the different sets of samples, with two miRNAs (hsa-miR-2467-5p and hsa-miR-4421) among the three different sets of comparisons being consistently found to be altered (Supplemental Figures S4-S7). 


\subsubsection{MiRNA Differences between Preeclampsia and Healthy Pregnancies}

We first explored ECFC by miRNA sequencing for differences in miRNA expression in the preeclampsia vs. control (healthy pregnancy) groups. This pairwise analysis was conducted independently for maternal blood and cord blood, as well as for P3 and P5. The levels of 17 miRNAs derived from cord blood at P3 were notably different between preeclampsia and control $(p<0.05$; Figure 1$)$. Four of those miRNAs additionally fulfilled the applied fold change criterion ( $>2$-fold), although none of them passed the BenjaminiHochberg correction at $p<0.05$ (Figure 1a). Comparing cord-blood-derived miRNA levels at P5, 47 miRNAs showed a notably different expression in the preeclampsia group $(p<0.05)$, but again none of these passed the Benjamini-Hochberg correction (Figure 1b). Using the same analysis for maternal blood samples, 39 miRNAs in P3 (Figure 1c) and 17 miRNAs in P5 (Figure 1d) were notably regulated. Again, none of these miRNAs passed the Benjamini-Hochberg correction. Heat maps of significantly regulated miRNAs in ECFC of controls compared to preeclampsia are shown in Supplemental Figure S2A. The most strongly regulated miRNAs passing the moderated $t$-test $(p<0.05$, fold change $>2)$ are shown in Table 2.
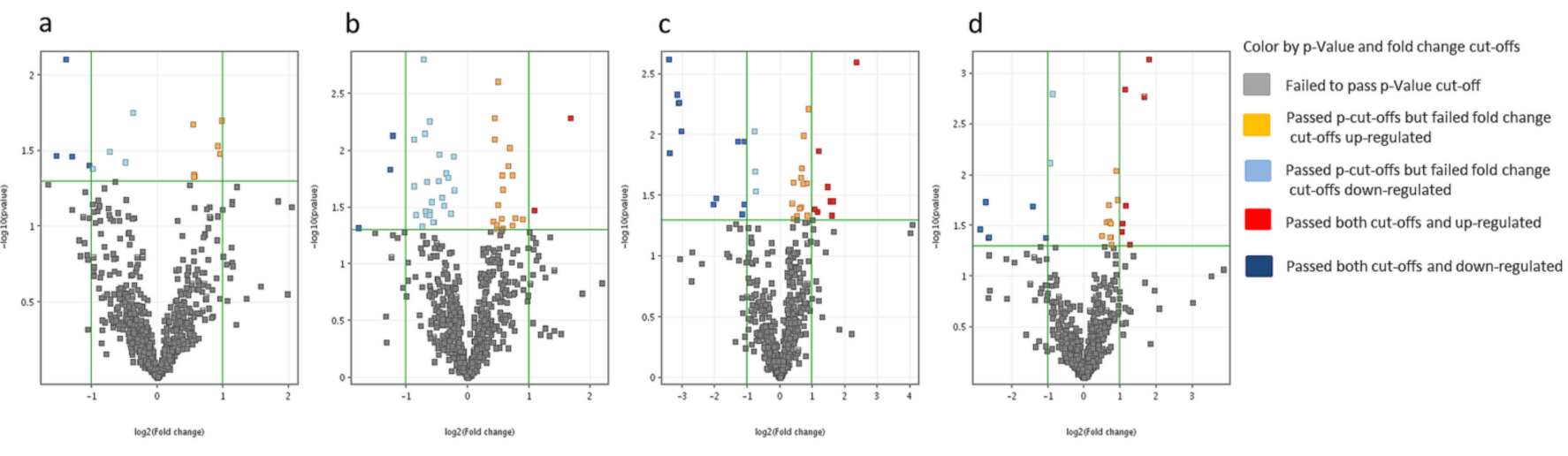

\begin{tabular}{lcccc}
\hline & \multicolumn{2}{c}{ Cord blood ECFC } & \multicolumn{2}{c}{ Maternal blood ECFC } \\
& Control vs. PE (P3) & Control vs. PE (P5) & Control vs. PE (P3) & Control vs. PE (P5) \\
\hline All Entities & 703 & 703 & 703 & 703 \\
$\begin{array}{l}\text { Moderated } \\
\text { t-test } \mathbf{p} \leq \mathbf{0 . 0 5}\end{array}$ & 17 & 47 & 39 & 17 \\
$\begin{array}{l}\text { Moderated t-test } \\
\mathbf{p} \leq \mathbf{0 . 0 5} \text { and FC }>\mathbf{2}\end{array}$ & 4 & 5 & 22 & 13 \\
Benjamini-Hochberg & 0 & 0 & 0 & 0
\end{tabular}

Figure 1. Significantly regulated miRNAs in ECFC of controls compared to preeclampsia subjects. Volcano plots show the fold changes of miRNAs on the $x$-axis and the statistical significance on the $y$-axis in relation to the reference group. Control: healthy pregnancy; PE: preeclamptic pregnancy; P3: passage 3; P5: passage 5; $n=6$ in each group. (a) Control vs. PE in P3 in cord-blood-derived ECFC. (b) Control vs. PE in P5 in cord-blood-derived ECFC. (c) Control vs. PE in P3 in maternal-blood-derived ECFC. (d) Control vs. PE in P5 in maternal-blood-derived ECFC.

\subsubsection{MiRNA Differences between Maternal and Cord Blood}

Next, we compared miRNA profiles between maternal blood and cord blood ECFC in P3 and P5 cells, with each divided into preeclampsia patients and controls (healthy pregnancies). This analysis resulted in 57 notably regulated miRNAs in P3 and 108 notably regulated miRNAs in P5 $(p<0.05)$ in ECFC derived from controls, with 4 of these miRNAs passing the Benjamini-Hochberg correction (Figure 2a,b). Comparison of miRNA profiles of maternal and cord blood ECFC derived from preeclampsia resulted in 78 notably regulated miRNAs in P3 and 163 notably regulated miRNAs in P5 $(p<0.05)$. Six miRNAs in P3 and 27 miRNAs in P5 passed Benjamini-Hochberg correction, respectively (Figure 2c,d). Heat 
maps of significantly regulated miRNAs in ECFC of cord blood compared to maternal blood are shown in Supplemental Figure S2B.

Table 2. Global overview of regulated miRNAs in preeclamptic ECFC vs. control ECFC with fold changes greater than 2 and $p$-values lower than 0.05 . Fold changes refer to the reference group (control =1).

\begin{tabular}{|c|c|c|}
\hline miRNA & Fold Change & $p$-Value \\
\hline \multicolumn{3}{|c|}{ ECFC from Cord blood in P3 } \\
\hline hsa-miR-4726-5p & -2.93 & 0.03 \\
\hline hsa-miR-1270 & -2.63 & 0.008 \\
\hline hsa-miR-148a-5p & -2.48 & 0.03 \\
\hline hsa-miR-1255a & -2.06 & 0.04 \\
\hline \multicolumn{3}{|c|}{ ECFC from Cord blood in P5 } \\
\hline hsa-miR-2467-5p & 3.19 & 0.005 \\
\hline hsa-miR-4687-3p & 2.11 & 0.03 \\
\hline hsa-miR-148a-3p & -3.44 & 0.04 \\
\hline hsa-miR-1226-5p & -2.40 & 0.02 \\
\hline hsa-miR-3911 & -2.34 & 0.007 \\
\hline \multicolumn{3}{|c|}{ ECFC from Maternal blood in P3 } \\
\hline hsa-miR-214-5p & -10.66 & 0.002 \\
\hline hsa-miR-199a-5p & -10.45 & 0.01 \\
\hline hsa-miR-214-3p & -8.89 & 0.005 \\
\hline hsa-miR-199a-3p & -8.52 & 0.005 \\
\hline hsa-miR-199b-3p & -8.52 & 0.005 \\
\hline hsa-miR-139-3p & -8.25 & 0.009 \\
\hline hsa-miR-139-5p & -4.13 & 0.04 \\
\hline hsa-miR-551a & -3.91 & 0.03 \\
\hline hsa-miR-4684-5p & -2.44 & 0.01 \\
\hline hsa-miR-3115 & -2.25 & 0.04 \\
\hline hsa-miR-3164 & -2.16 & 0.04 \\
\hline hsa-miR-199b-5p & -2.14 & 0.01 \\
\hline hsa-miR-4728-3 p & 5.06 & 0.003 \\
\hline hsa-miR-1250 & 3.10 & 0.04 \\
\hline hsa-miR-338-5p & 3.00 & 0.04 \\
\hline hsa-miR-338-3p & 2.92 & 0.04 \\
\hline hsa-miR-4485 & 2.75 & 0.03 \\
\hline hsa-miR-3177-5p & 2.27 & 0.01 \\
\hline hsa-miR-3128 & 2.21 & 0.04 \\
\hline hsa-miR-503 & 2.08 & 0.04 \\
\hline \multicolumn{3}{|c|}{ ECFC from Maternal blood in P5 } \\
\hline hsa-miR-214-5p & -7.34 & 0.03 \\
\hline hsa-miR-214-3p & -6.70 & 0.02 \\
\hline hsa-miR-199a-3p & -6.26 & 0.04 \\
\hline hsa-miR-326 & -2.70 & 0.02 \\
\hline hsa-miR-491-3p & -2.09 & 0.04 \\
\hline hsa-miR-4511 & 3.45 & 0.007 \\
\hline hsa-miR-3128 & 3.18 & 0.002 \\
\hline hsa-miR-4421 & 2.41 & 0.04 \\
\hline hsa-miR-2682-5p & 2.23 & 0.02 \\
\hline hsa-miR-3177-5p & 2.19 & 0.001 \\
\hline hsa-miR-5690 & 2.08 & 0.03 \\
\hline hsa-miR-2277-3p & 2.06 & 0.04 \\
\hline
\end{tabular}




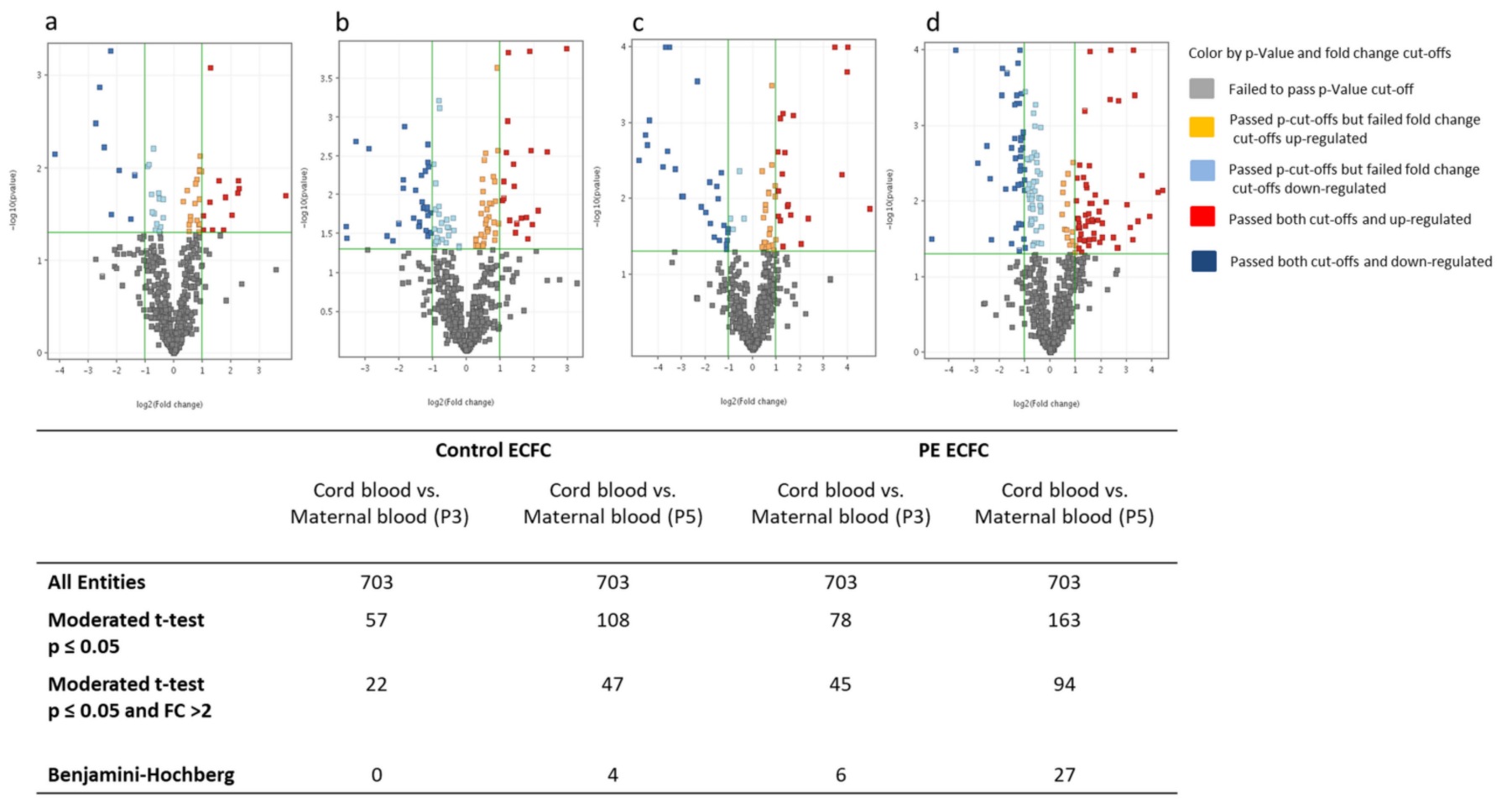

Figure 2. Significantly regulated miRNAs in ECFC of cord blood vs. maternal blood. Volcano plot shows the fold changes of miRNAs on the $x$-axis and the statistical significance on the $y$-axis in relation to the reference group. Control: healthy pregnancy; PE: preeclamptic pregnancy; P3: passage 3; P5: passage 5; $n=6$ in each group. (a) Control cord-blood-derived ECFC vs. maternal-blood-derived ECFC in P3. (b) Control cord-blood-derived ECFC vs. maternal-blood-derived ECFC in P5. (c) PE cord-blood-derived ECFC vs. maternal-blood-derived ECFC in P3. (d) PE cord-blood-derived ECFC vs. maternal-blood-derived ECFC in P5.

The most strongly regulated miRNAs that passed Benjamini-Hochberg correction can be found in Supplemental Table S1.

\subsubsection{MiRNA Differences between Cell Culture Passages 3 and 5}

Further, we asked whether differential miRNA levels remained stable and whether additional differences appeared with increasing cell passage. The comparison of the miRNA profiles of cord-blood-derived ECFC between P3 and P5 resulted in 19 notably regulated miRNAs derived from controls (healthy pregnancies) and 13 miRNAs in preeclampsia $(p<0.05)$. None of these miRNAs passed Benjamini-Hochberg correction (Figure $3 a, b)$. Comparison of miRNA profiles of maternal ECFC of P3 and P5 resulted in 10 (controls) and 5 (preeclampsia) notably regulated miRNAs. None of these miRNAs passed BenjaminiHochberg correction (Figure 3c,d). Heat maps of significantly regulated miRNAs in ECFC of P3 compared to P5 are shown in Supplemental Figure S2C.

A list of miRNAs with more than two-fold changes and significantly different levels $(p<0.05)$ between P3 and P5 is provided in Table 3. Interestingly, only one miRNA (hsamiR-3911) was shared between preeclampsia and healthy controls (control: fold change: $-2.16 ; p=0.007$; preeclampsia: fold change: $-21.26 ; p=0.02$ ), while 18 passage-associated miRNAs appeared as specific for the respective condition. 


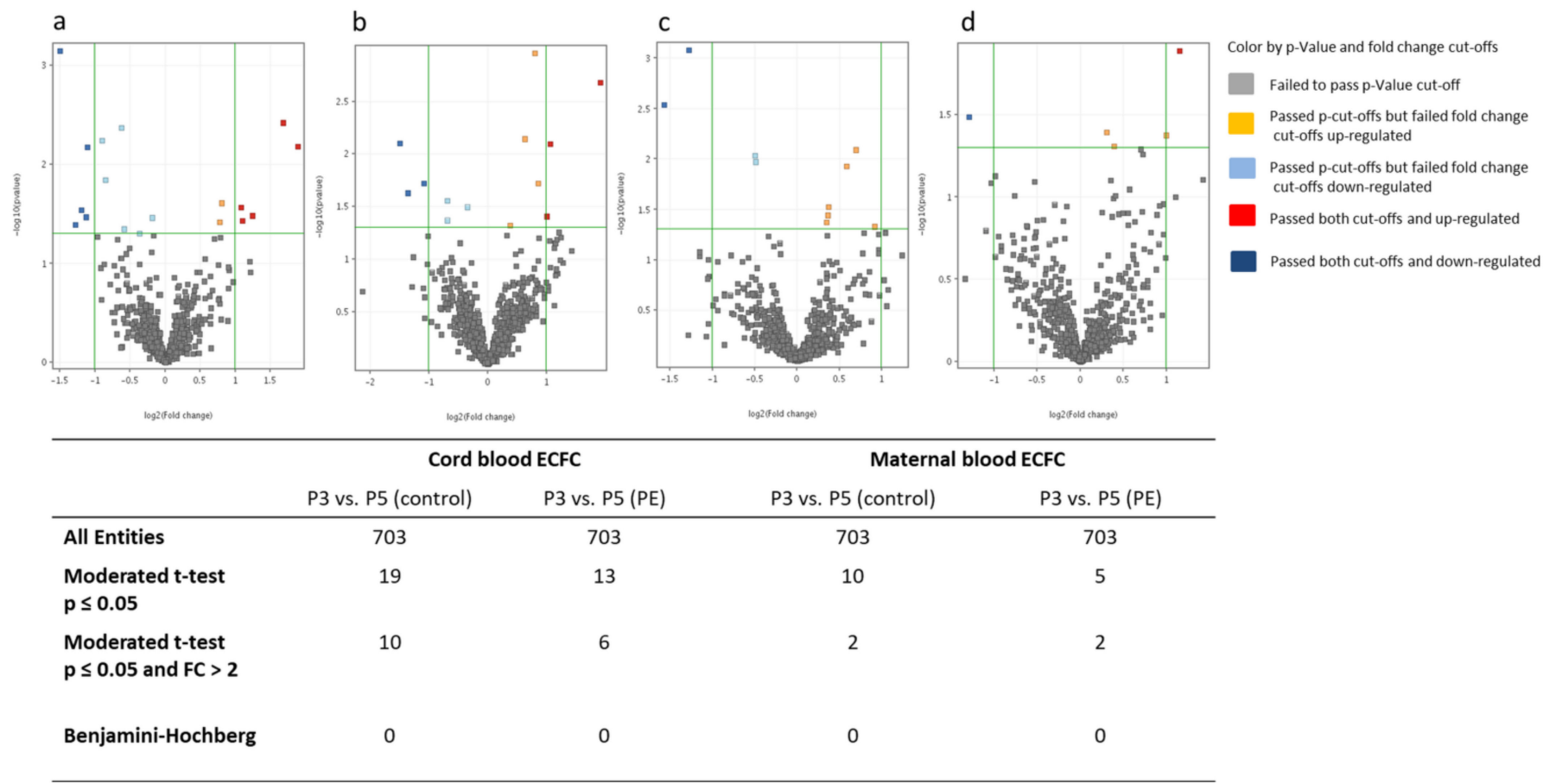

Figure 3. Significantly regulated miRNAs in ECFC of P3 vs. P5. Volcano plot shows the fold changes of miRNAs on the $\mathrm{x}$-axis and the statistical significance on the $\mathrm{y}$-axis in relation to the reference group. Control: healthy pregnancy; PE: preeclamptic pregnancy; P3: passage 3; P5: passage 5; $n=6$ in each group. (a) P3 vs. P5 in control cord blood ECFC. (b) P3 vs. P5 in PE cord blood ECFC. (c) P3 vs. P5 in control maternal blood ECFC. (d) P3 vs. P5 in PE maternal blood ECFC.

Table 3. Passage-associated differences in miRNA levels of ECFC from preeclampsia and controls with fold changes greater than 2 and $p$-values lower than 0.05 . Fold changes refer to the reference group $(\mathrm{P} 3=1)$.

\begin{tabular}{ccc}
\hline miRNA & Fold Change & $p$-Value \\
\hline \multicolumn{1}{c}{ Cord blood ECFC from controls in P3 vs. P5 } \\
\hline hsa-miR-4725-3p & -2.83 & \\
hsa-miR-1293 & -2.44 & 0.0007 \\
hsa-miR-3155a & -2.30 & 0.04 \\
hsa-miR-548s & -2.19 & 0.03 \\
hsa-miR-3911 & -2.16 & 0.03 \\
hsa-miR-451a & 3.70 & 0.007 \\
hsa-miR-122-5p & 3.20 & 0.007 \\
hsa-miR-5582-3p & 2.36 & 0.004 \\
hsa-miR-4726-5p & 2.14 & 0.03 \\
hsa-miR-2682-3p & 2.10 & 0.04 \\
\multicolumn{1}{c}{ Cord blood ECFC from preeclampsia in P3 vs. P5 } & 0.03 \\
\hline hsa-miR-1226-5p & -28.23 & \\
hsa-miR-5001-5p & -25.60 & 0.008 \\
hsa-miR-3911 & -21.26 & 0.02 \\
hsa-miR-3149 & 20.86 & 0.02 \\
hsa-miR-4791 & 20.16 & 0.008 \\
hsa-miR-2467-5p & 3.77 & 0.04 \\
\hline
\end{tabular}


Table 3. Cont.

\begin{tabular}{ccc}
\hline miRNA & Fold Change & $p$-Value \\
\hline & Maternal blood ECFC from controls in P3 vs. P5 \\
\hline hsa-let-7g-3p & -24.30 & 0.008 \\
hsa-miR-4421 & -2.97 & 0.003 \\
\hline \multicolumn{2}{c}{ Maternal blood ECFC from preeclampsia in P3 vs. P5 } \\
\hline hsa-miR-4677-5p & -2.45 & 0.03 \\
hsa-miR-4754 & 2.22 & 0.01 \\
\hline
\end{tabular}

\subsection{Quantitative Real-Time PCR Validation and Putative Target Gene Levels}

The profiles of the miRNAs with the most significant differences (lowest $p$-value) between preeclampsia and the control (healthy pregnancies) group, namely hsa-miRNA1270 (P3: $p=7.84 \times 10^{-3}$, fold change: -2.63 ) and hsa-miR-2467-5p (P5: $p=5.21 \times 10^{-3}$, fold change: 3.19 ) in cord-blood-derived ECFC, and hsa-miR-214-5p (P3: $p=2.38 \times 10^{-3}$, fold change: -10.66 ) and hsa-miR-3177-5p (P5: $p=1.44 \times 10^{-3}$, fold change: 2.19 ) in maternal-blood-derived ECFC, were further validated. QRT-PCR findings confirmed the results obtained by RNA sequencing for all four tested miRNAs (Figure 4a-d).
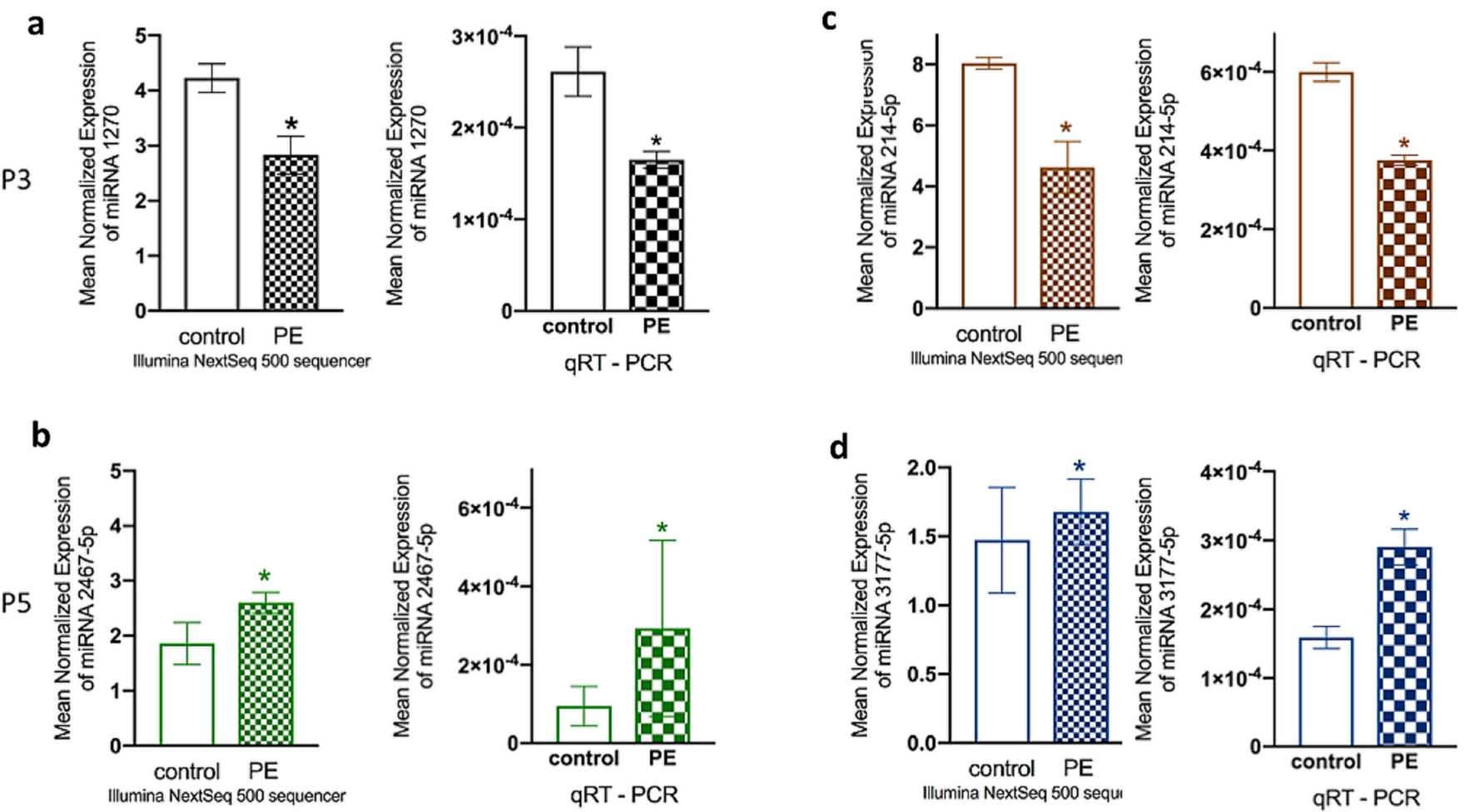

Cord blood

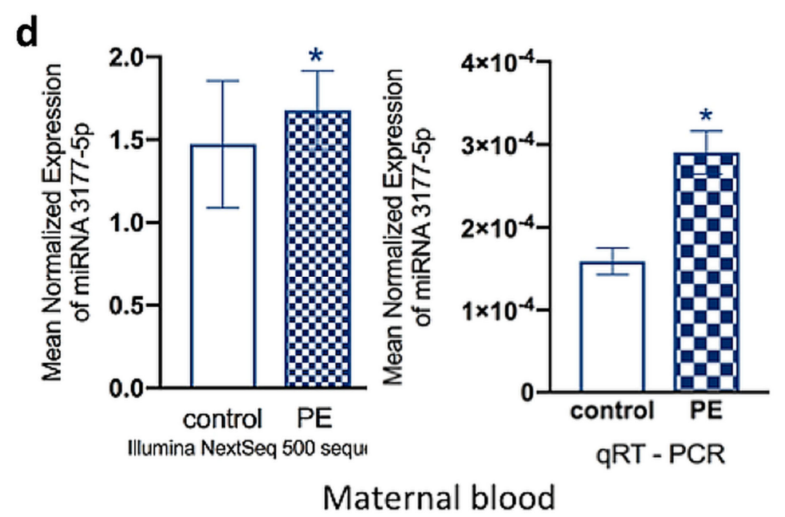

Figure 4. RNA sequencing and qRT-PCR results for hsa-miR-1270 (cord blood; P3) (a), hsa-miR-2467-5p (cord blood; P5) (b), hsa-miR-214-5p (maternal blood; P3) (c), and hsa-miR-3177-5p (maternal blood; P5) (d). Each RNA sample was validated in triplicate, and the relative expression level of each gene was normalized to that of RNU6, which served as the housekeeping gene $(n=6) .{ }^{*} p<0.05$.

Several genes have been reported as putative targets for the aforementioned miRNAs and are shown in Supplemental Table S2. Since we did not find much difference in miRNA profiles between P3 and P5, we chose to study only P3 cells for deeper validation, as they most closely resemble the phenotype of freshly isolated cells, and therefore are more comparable to cells in vivo than primary cells in P5. We selected NOSTRIN among the target genes of hsa-miR-214-5p because it has already been shown that NOSTRIN is 
increased in placental tissues of preeclamptic patients [20]. Since we could not detect any differences in the analysis of NOSTRIN hsa-miR-214-5p between preeclampsia vs. control (NOSTRIN: preeclampsia vs. control: $1.8 \times 10^{-6}$ vs. $1.0 \times 10^{-6} ; p=0.07$ ) (Figure $5 \mathrm{a}$ ), we analyzed target genes for hsa-miR-1270 (ANGPTL7 and TFRC). TFRC was chosen because it gave the highest score for hsa-miR-1270 on TargetScan and ANGPTL7 was selected because it is related to angiogenesis, and thus has an important link to preeclampsia. We selectively explored whether the levels of ANGPTL7 or TFRC correlated with hsa-miR-1270 in cord blood ECFC. We found significantly higher ANGPTL7 and TFRC mRNA levels in cord blood ECFC from preeclampsia compared to control (ANGPTL7: preeclampsia vs. control: $1.1 \times 10^{-5}$ vs. $5.7 \times 10^{-6} ; p=0.02$, Figure 5b; TFRC: preeclampsia vs. control: $1.9 \times 10^{-7}$ vs. $3.8 \times 10^{-8} ; p=0.009$, Figure 5 c).
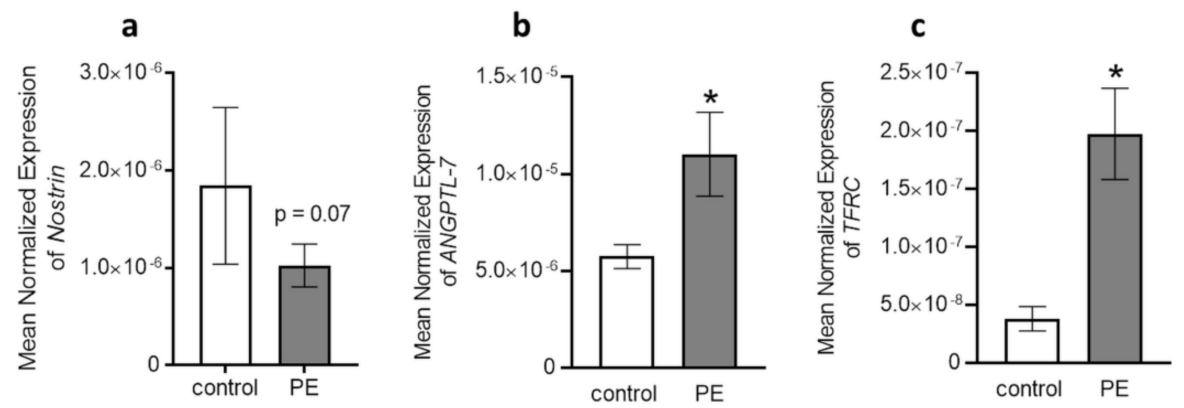

Figure 5. Transcript levels of predicted hsa-miR-214-5p target gene NOSTRIN (a) and hsa-miR-1270 target genes ANGPTL7 (b) and TFRC (c) in ECFC at passage 3 from control vs. preeclampsia (PE). Note: ${ }^{*} p<0.05, n=6$ per group. Each mRNA sample was validated in triplicate and the relative expression level for each was normalized to that of $18 \mathrm{~S}$.

\subsection{Pathway Enrichment Analysis}

To gain more insight into possible biological functions regulated by the miRNA that displayed the most differential abundance between preeclamptic and healthy pregnancies, we investigated the whole set of predicted targets using pathway enrichment analyses. First, we determined the set of predicted targets for the miRNA using TargetScan v.7.2 and miRDB, respectively. To reduce the number of false positives, we then subjected only those miRNA targets to further pathway analyses that were shared by both prediction tools (Figure 6 and Supplemental Table S2).
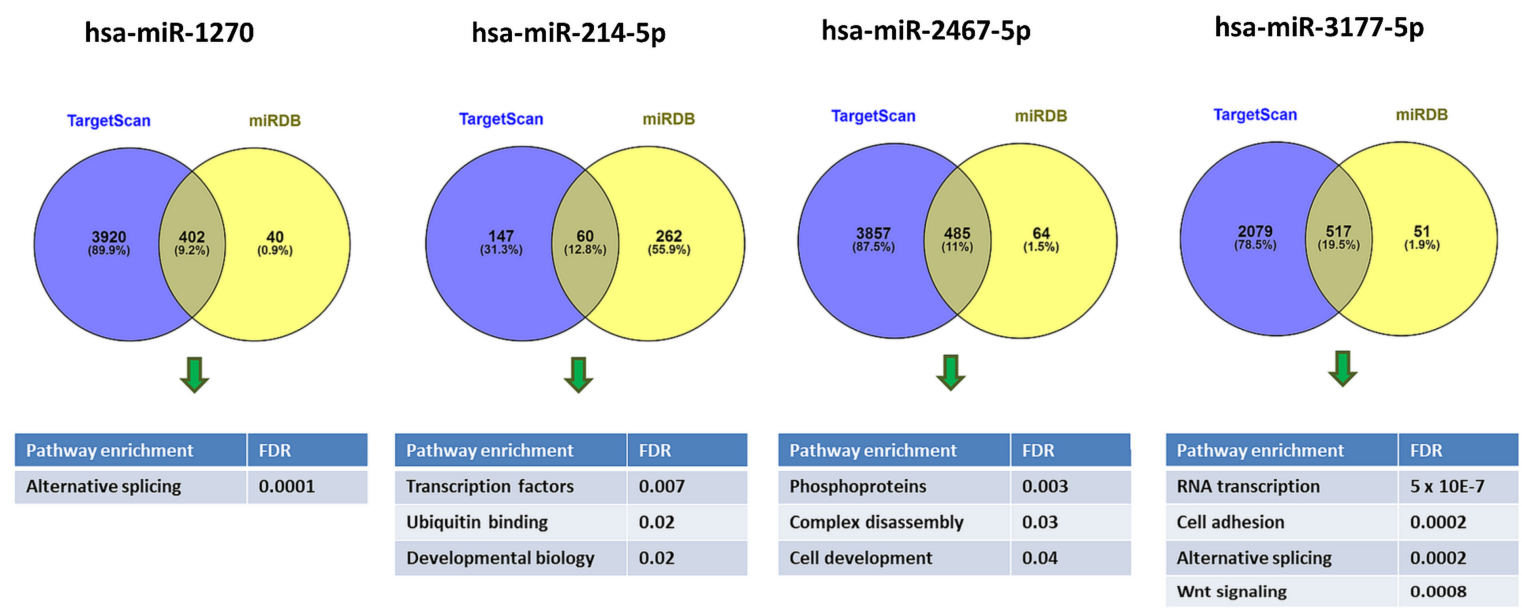

Figure 6. Venn diagram showing the overlap between predicted targets for hsa-miR-1270, hsa-miR-3177-5p, hsa-miR-214-5p, and hsa-miR-2467-5p that were shared by both TargetScan and miRDB prediction tools and were fed into further pathway analyses. Figure generated using Venny 2.1.0 (http:/ / bioinfogp.cnb.csic.es/tools/venny (accessed on 24 July 2019)). 
TargetScan predicted 4322 transcripts with sites for hsa-miR-1270, while miRDB predicted 442 targets, of which 402 were in common with TargetScan (Supplemental Table S2). This common set of targets was significantly enriched in genes with the UniProt term alternative splicing $(K W-0025, F D R=0.00013)$ (Supplemental Table S2).

We performed similar analyses with the top-ranking miRNAs from three other comparison groups of preeclampsia vs. control samples (Table 2). The hsa-miR-2467-5p had 485 predicted targets shared by TargetScan and miRDB that were notably enriched in phosphoproteins $(\mathrm{KW}-597, \mathrm{FDR}=0.007)$ and the GO term regulation of cell development (GO:0060284, FDR $=0.04)$. The hsa-miR-214-5p had 60 predicted targets shared by both TargetScan and $\mathrm{miRDB}$, but these were enriched in transcription factors (GO:0044798, FDR $=0.007)$. Finally, the hsa-miR-3177-5p had 517 predicted targets shared by TargetScan and miRDB, which were markedly enriched for RNA transcription (KW-0804, FDR $=5 \times 10^{-7}$ ). Additional significantly enriched pathways for hsa-miR-3177-5p included cell adhesion, alternative splicing, and Wnt signaling (Figure 6, Supplemental Table S2).

\subsection{Hsa-miR-1270 Dependent Tube Formation, Proliferation and Chemotactic Motility}

Endothelial cell function was assessed by a tube formation assay (Figure 7), which assessed the capability of endothelial cells to form capillary-like structures. ECFC were transfected with a hsa-miR-1270 inhibitor to mimic the observed down-regulation in ECFC. The specific hsa-miR-1270 inhibitor is a small specific RNA molecule that binds and inhibits hsa-miR-1270. Furthermore, a positive control for transfection efficiency was also included, which leads to an increase in HMGA2 in the qRT-PCR (Supplemental Figure S3). A negative control and a vehicle control (transfection reagent alone) were used to identify non-specific off-target effects. We examined whether hsa-miR-1270, which exhibited the greatest fold change in preeclampsia vs. control in cord-blood-derived cells, affects endothelial cell function. All ECFC were able to form tubes. Hsa-miR-1270 inhibition significantly reduced the total tube lengths between the groups (untreated control: $1.64 \times 10^{7} \mu \mathrm{m} \pm 1.8 \times 10^{6} \mu \mathrm{m}$; negative control: $1.63 \times 10^{7} \mu \mathrm{m} \pm 1.3 \times 10^{6} \mu \mathrm{m}$; hsa-miR-1270 inhibitor: $1.39 \times 10^{7} \mu \mathrm{m}$ $\pm 2.4 \times 10^{6} \mu \mathrm{m}$; untreated control vs. miR-1270 inhibitor: $p=0.028$; negative control vs. hsa-miR-1270 inhibitor: $p=0.025$ ) (Figure 7b). Transfection with hsa-miR-1270 inhibitor did not significantly change the number of formed loops as compared with the negative control (untreated control: $51.0 \pm 10.8$; negative control: $55.0 \pm 11.6$; hsa-miR-1270 inhibitor: $47.9 \pm 11.5$; untreated control vs. hsa-miR-1270 inhibitor: $p=0.49$; negative control vs. hsamiR-1270 inhibitor: $p=0.24$ ) (Figure 7a). The total branching points tended to decrease after transfection as compared with control but the difference was not significant (untreated control: $50.6 \pm 13.9$; negative control: $53.6 \pm 8.3$; hsa-miR-1270 inhibitor: $45.6 \pm 11.3$; untreated control vs. hsa-miR-1270 inhibitor: $p=0.44$; negative control vs. hsa-miR-1270 inhibitor: $p=0.13$ ) (Figure 7c).

Hsa-miR-1270 inhibition did not significantly change proliferation of ECFC in vitro as compared with control ECFC (population doubling time: untreated: $18.35 \mathrm{~h}$; negative control: $17.92 \mathrm{~h}$; hsa-miR-1270 inhibitor: $17.81 \mathrm{~h} ; p=0.32$ ) (Figure 8a), however the chemotactic motility levels of miR-1270-inhibited ECFC were impaired compared to negative or untreated control (Figure $8 \mathrm{~b}$ ) (untreated control: 84.1 cells/area; negative control: 64 cells/area; hsa-miR-1270 inhibitor: 48.6 cells/area; untreated control vs. miR-1270 inhibitor: $p=0.003$; negative control vs. hsa-miR-1270 inhibitor: $p=0.25$ ). 


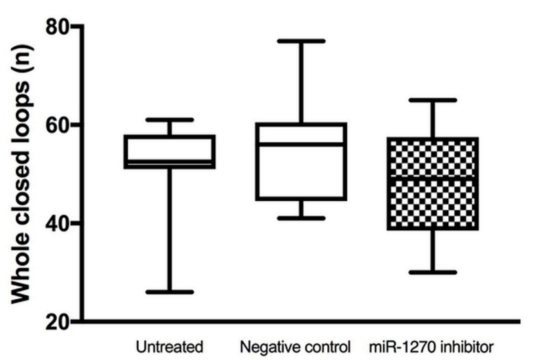

d Untreated

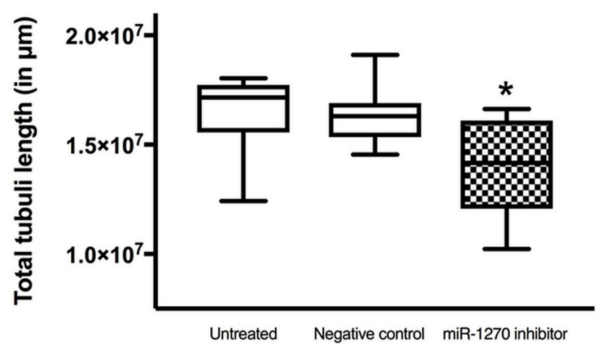

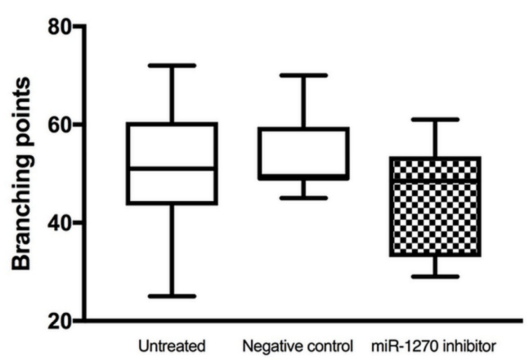

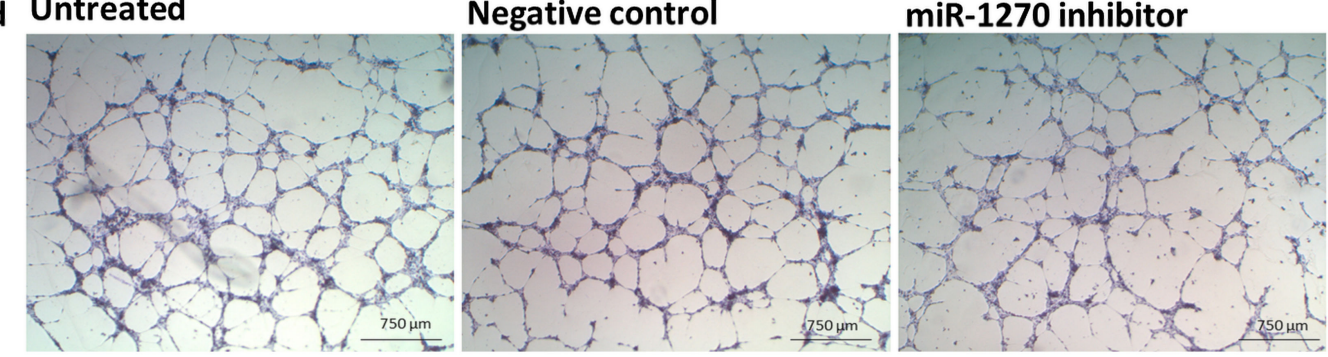

Figure 7. Tube formation of ECFC after inhibition of hsa-miR-1270: number of whole formed loops (a); total measured tubule length (b); total branching points (c); representative microphotographs (d); ${ }^{*} p<0.05, n=8$ per group. Scale bar $=750 \mu \mathrm{m}$.

a

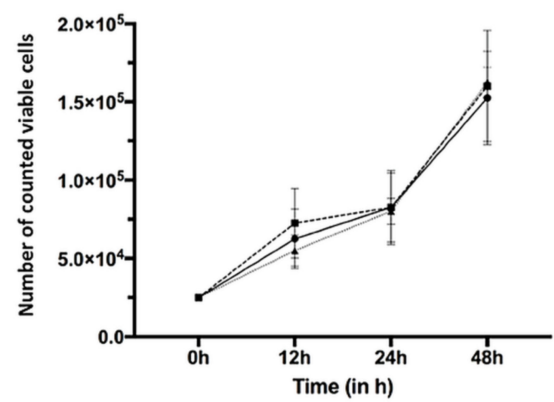

b

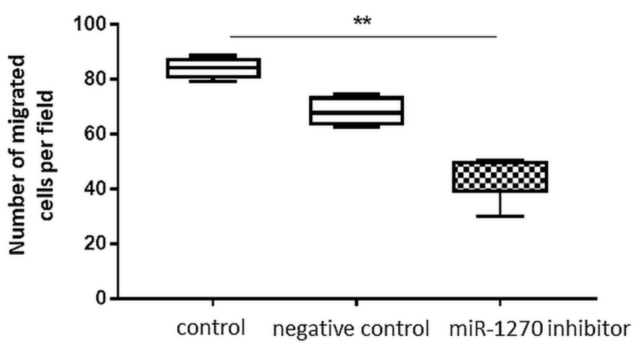

control negative control miR-1270 inhibitor

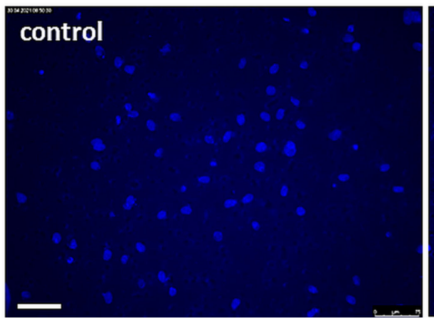

- control

-.- negative control

- miR-1270 inhibitor
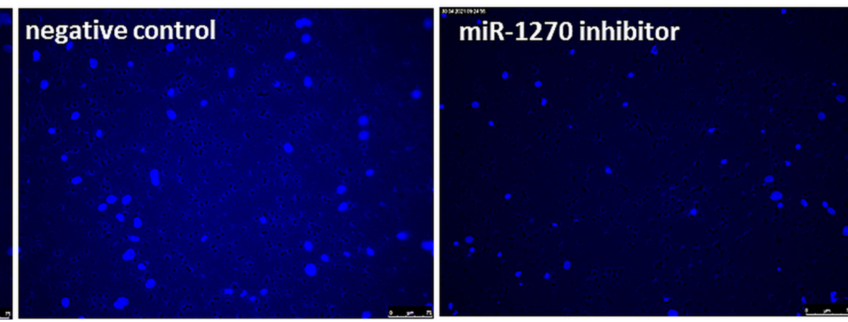

Figure 8. Proliferation (a) and chemotactic motility (b) of ECFC after inhibition of hsa-miR-1270 compared with negative control. Proliferation: Numbers of counted viable cells at 12,24 , and 48 h; $n=4$ per group. Chemotactic motility: Numbers of DAPI-stained migrated cells per area were counted in 5 randomly taken pictures from two different biological replicates. Experiments were performed in at least $2-3$ technical replicates; ${ }^{* *} p<0.01$. Scale $=75 \mu \mathrm{m}$.

\section{Discussion}

To our knowledge, this is the first study demonstrating that maternal-blood- and cord-blood-derived endothelial progenitor cells from preeclamptic pregnancies display an aberrant miRNA profile compared to healthy pregnancies. In this work, we focused on hsa-miR-1270, as it was one of the most statistically different miRNAs, with a high fold change in cord-blood-derived EFCF from preeclamptic vs. healthy mothers.

MiRNA studies of human-pregnancy-related tissues and cells have started to reveal important insights into pathogenesis, however whether aberrant miRNA patterns are 
involved in the functional changes observed in ECFC from preeclamptic pregnancies and if these lead to an increased cardiovascular risk are factors that have not been investigated. Previous studies on plasma and placental miRNAs have indicated the involvement of miRNAs in the pathophysiology of preeclampsia [21,22]. Although some reports [23-27] have already revealed the potential usefulness of measuring certain miRNAs for predicting the occurrence of preeclampsia, there have been few overlaps among those studies, and more investigation is required in order to clarify the exact role of preeclampsia-related miRNAs. This also applies to our study, in which two miRNAs (miR-139-5p, miR-148a) were detected that were reported to be down-regulated in other studies in preeclampsia but investigated in different tissues (placenta, decidua-derived mesenchymal stem cells) [28,29].

In our study, 17 miRNAs in P3 and 47 miRNAs in P5 showed a significantly different representation for cord-blood-derived preeclamptic ECFC when compared to control. The smallest $p$-value here was obtained for hsa-miR-1270, although this difference did not survive correction for multiple testing. In maternal ECFC, we found 39 differentially represented miRNAs in P3 and 17 in P5. Considering the therapeutic potential of ECFC, an essential aspect of our research was also the comparative analysis of two cell culture passages of the progenitor cells. P3 reflects the first passage after cell isolation that provides the required quantity and quality of ECFC for this and other analyses. Since primary cells are subject to aging, which is increasingly observed in higher passages, e.g., 7-8 onwards, we deliberately chose $\mathrm{P} 5$ as a comparison to avoid possible developmental changes. Here, we observed passage-associated changes in miRNA levels that were different in preeclampsiaderived ECFC compared with control ECFC, with only hsa-miR-3911 shared between both conditions. These observations suggest that there may be disease-associated changes affecting the propagation of preeclamptic ECFC in cell culture. The information gained is important, as it suggests that the priming of ECFC persists over cell culture passages and developmental effects play a minor role at least up to P5. Among the three different set of comparisons, hsa-miR-2467-5p and hsa-miR-4421 were consistently found to be altered among all of these comparisons.

Hsa-miR-1270 showed the smallest $p$-value in the preeclampsia group compared to the control. These results were further validated in vitro and putative target genes were investigated. The reduced level of miRNA hsa-miR-1270 observed by RNA sequencing in cord blood ECFC was confirmed by qRT-PCR. Interestingly, some predicted target gene products are also associated with preeclampsia, including the transferrin receptor, TFRC. TFRC is necessary for cellular iron uptake and has previously been noted to have markedly reduced expression in placentae from preeclamptic pregnancies compared to those from healthy pregnancies [30]. The level of TFRC mRNA was found to be significantly different in preeclampsia samples in our study, suggesting that TFRC might be regulated under steady-state conditions by hsa-miR-1270 in ECFC. A similar although less pronounced effect was observed for ANGPTL7.

According to our knowledge, only two studies have so far investigated the functional role of hsa-miR-1270, with conflicting results. While down-regulation of hsa-miR-1270 in papillary thyroid cancer cell lines TPCI and K1 suppressed cell proliferation, migration, and in vivo transplantation [31], up-regulation of hsa-miR-1270 suppressed human glioblastoma cancer cell proliferation, migration, and tumorigenesis [32]. Limited data exist on the effects of siRNA-mediated inhibition of TFRC and ANGPTL7. Increase of VEGF was observed in TFRC knockdown MDA-MB-231 cells when compared to the parental cells. In human primary human trabecular network cells, down-regulation of ANGPTL7 leads to significantly lower levels of extracellular matrix protein MMP1 but increases in fibronectin, collagen type VA1, myocilin, and versican [33]. As there are a magnitude of possible mechanisms and targets (see discussion of pathway enrichment analysis below), further investigation into the potential role of hsa-miR-1270 is required.

In a more global approach and through gene set enrichment analysis, we evaluated whether the sets of predicted targets of hsa-miR-1270 (and top-ranking miRNAs for the three other comparisons) mark specific pathways and protein families, which may be im- 
plicated in preeclampsia. The targets for hsa-miR-1270 and hsa-miR-3177-5p were enriched for alternative splicing, indicating that they might regulate gene expression. Alternative splicing of certain proteins has been implicated previously in the pathophysiology of preeclampsia, with the most notable example being s-Flt (for a review see Palmer et al., 2017 [34]). However, there is also evidence of a more global dysregulation of alternative splicing in preeclampsia [35]. Consistent with a role of the differentially represented miRNAs in gene expression, further enrichments were observed for RNA transcription and developmental pathways.

Our analyses were limited to only the top-ranking miRNAs, because none of the miRNAs passed the strict Benjamini-Hochberg correction. We extended our observations by a mechanistic approach and demonstrated that preeclampsia-specific miRNAs, e.g., hsa$\mathrm{miR}-1270$, affect endothelial progenitor cell angiogenic function in vitro. We acknowledge that the in vitro transfection used in our study differs from the situation in vivo. However, using miRNA inhibitors to analyze endogenous miRNA function is widely accepted as a tool to study the effects of different miRNA levels [36]. Although we observed a significant effect of hsa-miR-1270 inhibition on ECFC tubule length and chemotactic motility, no significant difference was noted in cell doubling. This effect seems biologically plausible. Compared to cell proliferation, tubule formation is a biologically more sophisticated process that requires a coordinated interaction between cells. Limitations of this complicated process may be of consequence much earlier. While hsa-miR-1270 seems to affect one part of the angiogenic process, further work will be needed to elucidate how the dysregulation of other identified miRNAs impact functional processes of EPC. Furthermore, we analyzed just one miRNA for functional differences in this study. A large-scale analysis such as luciferase binding assays in a larger study population would be useful to investigate miRNA-mediated regulation of target genes more specifically in preeclampsia. Our sample was limited to six cases per group, which is due to the well-described low isolation efficiency of ECFC. However, other publications on circulating [37] and ECFC-derived miRNAs [38] in pregnant populations provided new and interesting results, even with lower sample numbers. In addition, the comparison of mother-child pairs in both study populations would be desirable, as this would provide more stable conditions and avoid possible bias. While in our control group we were able to isolate ECFC from three mother-child pairs, none were available in the preeclampsia group. Regardless, the comparison of maternal and cord blood ECFC is a good starting point that should be expanded on in the future.

Nowadays, it is assumed that preeclampsia is essentially a disease of the vascular endothelium, which in turn contributes to an increased cardiovascular risk in later life. Discovered in the late 1990s, EPCs attracted clinical and basic research as key regulators of vascular homeostasis in health and disease, with their ability for neovasculogenesis, angiogenesis, and endothelial repair [8,39]. Since the first discovery of miRNAs, their involvement in different aspects of vascular disease has emerged as an important research field. MiRNAs have been found to be critical modulators of endothelial homeostasis, while dysregulation of miRNAs has been linked to endothelial dysfunction and the development and progression of vascular disease. Furthermore, insufficient angiogenesis is characterized by ischemic heart disease, peripheral vascular disease, and preeclampsia $[40,41]$. The relevance of miRNAs in vascular neovascularization has been demonstrated by different approaches of knockdown enzymes involved in the biogenesis of miRNAs [42-44]. As miRNAs are known to be critical in fine-tuning and in maintaining the physiological balance of the vascular endothelium, they are targets for miRNA-based therapies via reprogramming of endothelial cells. Although the effects of these potential therapeutic agents for endothelial function remain to be properly assessed, it is of crucial importance to first identify the potentially pathogenic miRNAs, such as in this pilot study.

In summary, we identified differentially expressed miRNA profiles that allowed us to predict target genes and pathways in preeclampsia that may be involved in the pathogenesis of the disease. Considering the small patient cohort, the results can serve as a basis for follow-up studies with a larger sample and a more limited selection of candidates 
to avoid the disadvantage of multiple testing. Epigenetic modifications are one of the potential mechanisms, including aberrant miRNA expression, through which the exposure to an altered in utero environment translates into the development of chronic disease. Understanding the impact of preeclampsia on epigenetic mechanisms and response of ECFC to therapeutic strategies will be critical in evaluating the utility of therapies designed to target this cell population.

\section{Materials and Methods}

\subsection{Participants}

We recruited subjects under Ethical Approval Number 3254 (Hannover Medical School) from the Division of Obstetrics. After informed consent, pre-delivery maternal blood and cord blood were collected and ECFC of 12 preeclamptic pregnancies $(n=6$ from maternal; $n=6$ from cord blood with no maternal-cord blood pairs) and 9 healthy pregnancies ( $n=6$ from maternal; $n=6$ from cord blood with 3 maternal-cord blood pairs) were isolated. Healthy participants had no significant maternal or neonatal disease. All were singleton pregnancies matched for gestational age at delivery, maternal BMI, and maternal age. Preeclampsia was defined as new-onset hypertension $\geq 140 / 90 \mathrm{mmHg}$ on two or more occasions after 20 weeks gestation accompanied by proteinuria or other symptoms of organ dysfunction, e.g., liver or acute kidney dysfunction, neurological symptoms, hemolysis, thrombocytopenia, or fetal growth restriction [45]. Resting blood pressure levels were taken by a trained nurse using the oscillometric method and the appropriate cuff size.

\subsection{ECFC Isolation and Characterization}

Primary human ECFC were isolated, characterized, and cultivated as previously described by others and our own studies $[12,46,47]$. Briefly, maternal peripheral (PBMC) or venous cord blood mononuclear cells (CBMC) were isolated by density gradient centrifugation and PBMC or CBMC were plated $\left(5 \times 10^{7}\right.$ cells /well) onto collagen-coated 6-well plates (BD Bioscience, Heidelberg, Germany) containing endothelial growth medium 2 (EGM-2, Lonza, Basel, Switzerland), supplemented with the supplier's recommended concentrations of growth factors (10\% FBS and 1\% penicillin-streptomycin). After 10-21 days of cultivation, ECFC appeared as adherent single layers of cobblestone-shaped, late-outgrowth cells that formed colonies ( $>50$ cells). Flow cytometric analyses to confirm the ECFC phenotype were performed using surface markers CD31, CD133, and CD45, as well as appropriate isotype controls (Supplemental Figure S1).

\subsection{Small RNA Sequencing}

The global miRNA profiles of maternal-blood- and cord-blood-derived ECFC from six preeclamptic pregnancies and six uncomplicated, healthy pregnancies were determined by small RNA sequencing. For miRNA isolation, cultured ECFC were lysed on ice and RNA was extracted according to the recommended protocol variant for total RNA including small species (mirVANA Kit, Ambion, MA, USA). Library preparation for small RNA sequencing was performed by use of the NEBNext ${ }^{\circledR}$ Multiplex Small RNA Library Prep Kit for Illumina ${ }^{\circledR} 96$ rxns, Index Primers 1-48 (E7560S, New England Biolabs, Frankfurt am Main, Germany).

Forty-eight libraries (reflecting 48 biological samples) were specifically barcoded, pooled, denatured, and diluted. Two denatured pools, each containing 24 libraries, were run on an Illumina NextSeq 550 sequencer, using two high-output flow cells for $75 \mathrm{bp}$ single reads (San Diego, IL, USA) and yielding 15 million reads on average per analyzed sample (median: 13.5 million). Raw data processing and quality control were conducted with bcl2fastq Conversion Software, Trim Galore, and FastQC, while mapping, quantification, normalization, and differential expression analyses were performed with StrandNGS v3.1 (Avadis, Bangalore, Karnataka, India). Levels of 2104 miRNAs were considered in the analysis based on their read counts. In order to eliminate undetectable or poorly 
reliable miRNA signatures, data were positively filtered for miRNAs, showing at least two normalized read counts in all samples of at least one out of the eight analyzed groups, namely maternal- or cord-blood-derived, cell passage 3 or 5 , and control or preeclampsia. This first expression filter yielded 703 miRNAs that were deemed expressed, which served as a starting point for subsequent analyses. The Shapiro-Wilk test was used to investigate whether resulting expression data were distributed normally. For miRNAs with $p$-values greater than the chosen alpha level (0.01), we accepted the null hypothesis that the data came from a normally distributed population. These miRNAs were further analyzed using the moderated $t$-test $(p<0.05)$. In addition, $p$-values were corrected using the BenjaminiHochberg procedure to account for multiple testing and to estimate the false discovery rate (FDR). Normalized log-2-transformed count data were subsequently baseline transformed to the median. These data were imported into Omics Explorer v3.6 (Qlucore, Lund, Sweden) for final heatmap visualization. More detailed information on RNA sequencing can be found in Supplemental Methods S1.

MiRNA profiles of ECFC from preeclamptic and healthy pregnancies were compared. Separate analyses were performed in ECFC derived from cord blood (offspring group, $n=6$ ) and ECFC derived from maternal blood (maternal group, $n=6$ ). All analyses were performed at two cell culture passages, P3 and P5. In a second approach, we compared ECFC isolated from maternal blood with ECFC isolated from cord blood. Separate analyses were performed in ECFC derived from preeclampsia and healthy pregnancies (the control group). Again, these analyses were performed at two cell culture passages, P3 and P5. Thirdly, we examined whether cell culture passage affects miRNA patterns overall. P3 corresponded to the earliest time when sufficient cell numbers were available after primary cell isolation, while P5 was selected to fall within a common window where ECFC are used for functional studies [37,41,42]. Separate analyses were performed in ECFC derived from cord blood (offspring group) and ECFC derived from maternal blood (maternal group). In this approach, the analyses were stratified by disease status. For further validation and target gene expression analysis of maternal and cord blood miRNAs, we first focused on the biologically relevant effect sizes by filtering for miRNAs with log-fold changes $\geq 2$, followed by the most significant hit (lowest $p$ value), independent of its effect size, which is a common procedure in case-control association studies. For the final selection step, the highest fold change was considered.

\section{4. qRT-PCR for miRNA Validation and Putative Target Gene Expression}

To evaluate the miRNA level, an miScript Primer Assay (Qiagen, Leipzig, Germany, Cat. No.: MS00014392 (hsa-miR-1270)) was used according to the manufacturer's recommendations to convert RNA for to cDNA synthesis. The qRT-PCR was conducted with miScript II Master Mix (miScript II, SYBR Green Assay, Qiagen, Leipzig, Germany, 218160) on a Corbett Rotor Gene (Corbett Life Science, Sydney, Australia).

Putative target genes of differently represented miRNAs were predicted via TargetScan (http:/ /www.targetscan.org/vert_72/, accessed on 24 July 2019) [48] and further analyzed for mRNA level via qRT-PCR. For normalization, 18S rRNA (RNA18S1) served as the housekeeping gene.

Primer sequences for determination of mRNA and miRNA levels are described in Supplemental Table S3A,B. For qRT-PCR analysis of miRNA, the miScript Primer Assays with miRNA-specific forward primer (Supplemental Table S3B) and the miScript SYBR Green PCR Kit, containing the miScript Universal Primer (reverse primer) and QuantiTect SYBR Green PCR Master Mix, were used. Relative quantification of gene expression was calculated by standard $\triangle \mathrm{Ct}$ method using the expression of $18 \mathrm{~S}$ rRNA (target genes) or RNU6 (miRNA) as the reference. For each treatment and qRT-PCR analysis of miRNA and mRNA, runs were performed in triplicate. A total of three RT-PCR runs were performed for each patient. Relative levels in six ECFC from the respective groups were compared. 


\subsection{Transfection of ECFC with miRNA Inhibitor}

Down-regulation of hsa-miR-1270 was observed in our study. To analyze this effect in vitro, we used a miRNA inhibitor, a small RNA molecule that inhibits miRNA by binding. At $70-85 \%$ confluence, ECFC were transfected with either mirVana ${ }^{\mathrm{TM}}$ miR-1270 inhibitor (ID: MH13389), mirVana ${ }^{\mathrm{TM}}$ miRNA inhibitor let-7c positive control (\#4464080), mirVana ${ }^{\mathrm{TM}}$ miRNA inhibitor negative control \#1 (4464076) (Thermo Fisher Scientific, Waltham, MA, USA), or vehicle control (transfection reagent alone: Lipofectamine RNAiMAX, Invitrogen, Waltham, MA, USA) according to the manufacturer's protocol. The mirVana ${ }^{\mathrm{TM}}$ miRNA inhibitor let-7c positive control was used to determine transfection efficiency. After transfection into cells, the let-7c miRNA inhibitor blocks endogenous let-7c miRNA, leading to elevated HMGA2 mRNA levels, which are analyzed by qRT-PCR. The mirVana ${ }^{\mathrm{TM}}$ miRNA inhibitor let-7c positive control was transfected in parallel with the miR-inhibitor or mirVana ${ }^{\mathrm{TM}}$ miRNA inhibitor negative control \#1 (Supplemental Figure S3). For transfection, $9 \mu \mathrm{L}$ Lipofectamine RNAiMAX reagent was diluted in $150 \mu \mathrm{L}$ Opti-MEM Medium (Life Technologies, Waltham, MA, USA) and $30 \mathrm{nM}$ miRNA inhibitor or related controls, which were diluted in $150 \mu \mathrm{L}$ Opti-MEM medium. Cells were incubated for $24 \mathrm{~h}$ at $37^{\circ} \mathrm{C}$ and $5 \% \mathrm{CO}_{2}$ before they were used for functional assays. Transfection efficiency was determined via qRT-PCR by expression of HMGA2 mRNA (positive control). Then, $24 \mathrm{~h}$ after transfection, ECFC were analyzed for tube formation capacity and proliferation ability.

\subsection{Tube Formation Assay}

An in vitro angiogenesis assay was used to test the capacity of ECFC to form capillary tubule-like networks. Here, $1.7 \times 10^{4}$ cells/well of hsa-miR-1270-inhibitor-transfected ECFC were seeded in a 96-well plate containing treatment medium and growth-factorreduced Matrigel matrix (BD Biosciences, Bedford, MA, USA) and transferred onto a LEICA DMI 6000 B microscope equipped with Incubator BL (Leica, Wetzlar, Germany) for heating and $5 \% \mathrm{CO}_{2}$ supply. Images were taken by using phase contrast optics with a $2.5 \times$ objective after $12 \mathrm{~h}$. The total tubule length in each visual field, quantity of whole closed loops, and number of interconnections between the tubules (branching points) were measured using Image J software 1.52q (National Institutes of Health, Bethesda, MA, USA). The total tubule length describes the sum of all tubule fibers of the entire image section. The quantity of whole closed loops is the number of all closed tubular loops in the image section. Branching points were defined as points of interaction from which at least three tubules originated.

\subsection{Cell Proliferation Assay}

To determine the proliferative capacity of ECFC after inhibition of hsa-miR-1270, $2.5 \times 10^{4}$ cells were seeded per well of 24-well culture plates in EGM supplemented with $5 \%(v / v)$ FBS and 1\% penicillin-streptomycin. After 12, 24, and $48 \mathrm{~h}$, the cell number and population doubling time were determined by trypan blue staining. ECFC collected after $24 \mathrm{~h}$ of transfection and a corresponding untreated and negative control were run in tandem.

\subsection{Chemotaxis Assay}

Chemotaxis assay was performed by using transwell inserts with a translucent microporous membrane (ThinCerts, Greiner, Kremsmünster, Austria), as recently described [49]. After transfection with hsa-miR-1270 inhibitor or related controls, cells were serum-deprived for $24 \mathrm{~h}$ with growth medium supplemented with $2.5 \%$ FCS. After $24 \mathrm{~h}$, cells were trypsinized and $7 \times 10^{4}$ cells were seeded into the apical side of the insert in serum-free medium. As a chemoattractant, medium supplemented with $10 \%$ FCS was applicated in the basolateral side of the insert and cells were placed in an incubator for $4 \mathrm{~h}$. Non-migrated cells were removed with a cotton bud from the apical side of the insert, while migrated cells on the insert membrane were fixed in 3\% PFA, 2\% sucrose in PBS for $10 \mathrm{~min}$, washed two times with PBS, and counterstained with 4',6-diamidin-2-phenylindol ((DAPI) 
(Invitrogen, Waltham, MA, USA)). After mounting in antifade fluorescence mounting medium (ProLongGold, Thermo Fisher Scientific, Waltham, MA, USA), five pictures per condition were randomly taken and DAPI-stained cells per area were counted to analyze chemotactic migration.

\subsection{Statistics and Bioinformatics Analyses}

Data distribution was compared between groups using the Shapiro-Wilk test. Differences in the mean and median levels of continuous data were tested using Mann-Whitney $\mathrm{U}$ test or Wilcoxon two-sample test as appropriate. Categorical variables were compared by Fisher's exact test. All $p$-values were two-sided, and $p$-values less than 0.05 were considered statistically significant. Where multiple testing had to be considered, $p$-values less than 0.05 were considered noteworthy before FDR correction and considered statistically significant if they remained less than 0.05 after FDR correction.

We compared predicted targets for miRNAs with different levels of abundance in preeclampsia vs. healthy pregnancies at P3. Target predictions were accomplished using the Target Scan 7.2 database (http:/ / www.targetscan.org/vert_72) [48] and the miRDB database (http:/ / mirdb.org/) [50]. Target sets were then investigated using the database of the Gene Ontology (GO) Consortium (www.geneontology.org/) [51] for an enrichment of biological pathways as defined in the Kyoto Encyclopedia of Genes and Genomes (KEGG) database (www.genome.jp/kegg/) [52], and for predominant protein domains according to the PFAM database (https://pfam.xfam.org/) [53]. These resources are publicly accessible and are implemented in STRING (www.string-db.org/) [54]. All databases were accessed on 24 July 2019. Biological processes, pathways, or protein domains were considered significantly enriched after correction for multiple testing if the FDR was $<0.05$.

\section{Conclusions}

The data obtained are novel both in terms of the cells studied and the disease pathogenesis investigated. Furthermore, this study lays the foundation for future work investigating whether miRNA differences contribute to cardiovascular risk in women and offspring from pregnancies complicated by preeclampsia. Considering the potential of EPC-based cytotherapies, further studies on their miRNA modifications by complicated pregnancies are warranted to develop miRNA-based therapeutics targeting cardiovascular complications. An epigenetically modified endothelial precursor during embryo- and fetogenesis may affect both normal morphogenesis and post-natal physiological adult progenitor-mediated vascular repair. Therefore, the miRNA status of ECFC may be key to understanding the pathogenesis of cardiovascular changes after preeclampsia, as well as the ultimate long-term health of mother and offspring.

Supplementary Materials: The following are available online at https:/ /www.mdpi.com/article/10 .3390/ijms22105320/s1.

Author Contributions: Conceptualization, L.B., B.S.-H., and F.v.V.-H.; data curation, L.B., S.v.H., B.S.-H., T.D., and F.v.V.-H.; formal analysis, L.B. and S.v.H.; funding acquisition, F.v.V.-H. and T.D.; investigation, L.B., B.S.-H., S.v.H., K.R., and N.M.; methodology, O.D.-B., T.D., and F.v.V.-H.; resources, C.S.v.K. and F.v.V.-H.; software, O.D.-B. and T.D.; supervision, F.v.V.-H.; validation, L.B., S.v.H., T.D., and F.v.V.-H.; visualization, L.B. and T.D.; writing—original draft, L.B. and F.v.V.-H.; writing-review and editing, L.B., B.S.-H., T.D., and F.v.V.-H. All authors have read and agreed to the published version of the manuscript. 
Funding: This research was funded by The Boehringer Ingelheim Foundation with an Exploration Grant to Frauke von Versen-Höynck and a grant by the German Research Foundation to Frauke von Versen-Höynck (DFG VE 490/15-1) and Thilo Dörk (DO 761/17-1). We acknowledge support by the German Research Foundation (DFG) and the Open Access Publication Fond of Hannover Medical School (MHH).

Institutional Review Board Statement: The study was conducted according to the guidelines of the Declaration of Helsinki and approved by the Ethics Committee of Hannover Medical School (Ethical Approval Number 3254).

Informed Consent Statement: Informed consent was obtained from all subjects involved in the study.

Data Availability Statement: The data that support the findings of this study are available from the corresponding author upon reasonable request.

Acknowledgments: The authors thank the faculty, residents, and staff of the Division of Obstetrics at Hannover Medical School for support in recruiting participants and collecting blood samples.

Conflicts of Interest: The authors declare no conflict of interest. The funders had no role in the design of the study; in the collection, analyses, or interpretation of data; in the writing of the manuscript; or in the decision to publish the results.

\section{References}

1. Stojanovska, V.; Scherjon, S.A.; Plosch, T. Preeclampsia as modulator of offspring health. Biol. Reprod. 2016, 94, 53. [CrossRef]

2. Davis, E.F.; Lazdam, M.; Lewandowski, A.J.; Worton, S.A.; Kelly, B.; Kenworthy, Y.; Adwani, S.; Wilkinson, A.R.; McCormick, K.; Sargent, I.; et al. Cardiovascular risk factors in children and young adults born to preeclamptic pregnancies: A systematic review. Pediatrics 2012, 129, e1552-e1561. [CrossRef] [PubMed]

3. Fraser, A.; Nelson, S.M.; Macdonald-Wallis, C.; Sattar, N.; Lawlor, D.A. Hypertensive disorders of pregnancy and cardiometabolic health in adolescent offspring. Hypertension 2013, 62, 614-620. [CrossRef] [PubMed]

4. $\quad$ Brown, M.C.; Best, K.E.; Pearce, M.S.; Waugh, J.; Robson, S.C.; Bell, R. Cardiovascular disease risk in women with pre-eclampsia: Systematic review and meta-analysis. Eur. J. Epidemiol. 2013, 28, 1-19. [CrossRef] [PubMed]

5. Powers, R.W.; Catov, J.M.; Bodnar, L.M.; Gallaher, M.J.; Lain, K.Y.; Roberts, J.M. Evidence of endothelial dysfunction in preeclampsia and risk of adverse pregnancy outcome. Reprod. Sci. 2008, 15, 374-381. [CrossRef] [PubMed]

6. Schmidt-Lucke, C.; Rossig, L.; Fichtlscherer, S.; Vasa, M.; Britten, M.; Kamper, U.; Dimmeler, S.; Zeiher, A.M. Reduced number of circulating endothelial progenitor cells predicts future cardiovascular events: Proof of concept for the clinical importance of endogenous vascular repair. Circulation 2005, 111, 2981-2987. [CrossRef] [PubMed]

7. Werner, N.; Kosiol, S.; Schiegl, T.; Ahlers, P.; Walenta, K.; Link, A.; Bohm, M.; Nickenig, G. Circulating endothelial progenitor cells and cardiovascular outcomes. N. Engl. J. Med. 2005, 353, 999-1007. [CrossRef]

8. Asahara, T.; Murohara, T.; Sullivan, A.; Silver, M.; van der Zee, R.; Li, T.; Witzenbichler, B.; Schatteman, G.; Isner, J.M. Isolation of putative progenitor endothelial cells for angiogenesis. Science 1997, 275, 964-967. [CrossRef]

9. King, T.F.; McDermott, J.H. Endothelial progenitor cells and cardiovascular disease. J. Stem Cells 2014, 9, 93-106.

10. Luppi, P.; Powers, R.W.; Verma, V.; Edmunds, L.; Plymire, D.; Hubel, C.A. Maternal circulating CD34+VEGFR-2+ and CD133+VEGFR-2+ progenitor cells increase during normal pregnancy but are reduced in women with preeclampsia. Reprod. Sci. 2010, 17, 643-652. [CrossRef]

11. Lin, C.; Rajakumar, A.; Plymire, D.A.; Verma, V.; Markovic, N.; Hubel, C.A. Maternal endothelial progenitor colony-forming units with macrophage characteristics are reduced in preeclampsia. Am. J. Hypertens. 2009, 22, 1014-1019. [CrossRef]

12. Von Versen-Hoynck, F.; Brodowski, L.; Dechend, R.; Myerski, A.C.; Hubel, C.A. Vitamin D antagonizes negative effects of preeclampsia on fetal endothelial colony forming cell number and function. PLoS ONE 2014, 9, e98990. [CrossRef]

13. Brodowski, L.; von Hardenberg, S.; Schröder-Heurich, B.; von Kaisenberg, C.S.; Hubel, C.A.; Dörk, T.; von Versen-Höynck, F. Preeclampsia-associated alteration of dna methylation in fetal endothelial progenitor cells. Front. Cell. Dev. Biol. 2019, 7, 1-14. [CrossRef]

14. Ambros, V. The functions of animal microRNAs. Nature 2004, 431, 350-355. [CrossRef]

15. Rosenfeld, N.; Aharonov, R.; Meiri, E.; Rosenwald, S.; Spector, Y.; Zepeniuk, M.; Benjamin, H.; Shabes, N.; Tabak, S.; Levy, A.; et al. MicroRNAs accurately identify cancer tissue origin. Nat. Biotechnol. 2008, 26, 462-469. [CrossRef]

16. Santulli, G. microRNAs and endothelial (Dys) function. J. Cell Physiol. 2015. [CrossRef]

17. Colpaert, R.M.W.; Calore, M. MicroRNAs in cardiac diseases. Cells 2019, 8, 737. [CrossRef]

18. Mellis, D.; Caporali, A. MicroRNA-based therapeutics in cardiovascular disease: Screening and delivery to the target. Biochem. Soc. Trans. 2018, 46, 11-21. [CrossRef]

19. Takahashi, Y.; Satoh, M.; Minami, Y.; Tabuchi, T.; Itoh, T.; Nakamura, M. Expression of miR-146a/b is associated with the Toll-like receptor 4 signal in coronary artery disease: Effect of renin-angiotensin system blockade and statins on miRNA-146a/b and Toll-like receptor 4 levels. Clin. Sci. 2010, 119, 395-405. [CrossRef] 
20. Xiang, W.; Chen, H.; Xu, X.; Zhang, M.; Jiang, R. Expression of endothelial nitric oxide synthase traffic inducer in the placentas of women with pre-eclampsia. Int. J. Gynaecol. Obstet. 2005, 89, 103-107. [CrossRef]

21. Lv, Y.; Lu, C.; Ji, X.; Miao, Z.; Long, W.; Ding, H.; Lv, M. Roles of microRNAs in preeclampsia. J. Cell Physiol. 2019, $234,1052-1061$. [CrossRef]

22. Apicella, C.; Ruano, C.S.M.; Méhats, C.; Miralles, F.; Vaiman, D. The role of epigenetics in placental development and the etiology of preeclampsia. Int. J. Mol. Sci. 2019, 20, 2837. [CrossRef] [PubMed]

23. Hromadnikova, I.; Kotlabova, K.; Doucha, J.; Dlouha, K.; Krofta, L. Absolute and relative quantification of placenta-specific micrornas in maternal circulation with placental insufficiency-related complications. J. Mol. Diagn. 2012, 14, 160-167. [CrossRef] [PubMed]

24. Hromadnikova, I.; Kotlabova, K.; Ondrackova, M.; Kestlerova, A.; Novotna, V.; Hympanova, L.; Doucha, J.; Krofta, L. Circulating C19MC microRNAs in preeclampsia, gestational hypertension, and fetal growth restriction. Mediat. Inflamm. 2013, $2013,186041$. [CrossRef]

25. Gunel, T.; Zeybek, Y.G.; Akcakaya, P.; Kalelioglu, I.; Benian, A.; Ermis, H.; Aydinli, K. Serum microRNA expression in pregnancies with preeclampsia. Genet. Mol. Res. 2011, 10, 4034-4040. [CrossRef] [PubMed]

26. Yang, Q.; Lu, J.; Wang, S.; Li, H.; Ge, Q.; Lu, Z. Application of next-generation sequencing technology to profile the circulating microRNAs in the serum of preeclampsia versus normal pregnant women. Clin. Chim. Acta Int. J. Clin. Chem. 2011, 412, 2167-2173. [CrossRef] [PubMed]

27. Zhang, Y.; Fei, M.; Xue, G.; Zhou, Q.; Jia, Y.; Li, L.; Xin, H.; Sun, S. Elevated levels of hypoxia-inducible microRNA-210 in pre-eclampsia: New insights into molecular mechanisms for the disease. J. Cell. Mol. Med. 2012, 16, 249-259. [CrossRef] [PubMed]

28. Enquobahrie, D.A.; Abetew, D.F.; Sorensen, T.K.; Willoughby, D.; Chidambaram, K.; Williams, M.A. Placental microRNA expression in pregnancies complicated by preeclampsia. Am. J. Obstet. Gynecol. 2011, 204, 178.e12-178.e21. [CrossRef]

29. Zhao, G.; Zhou, X.; Chen, S.; Miao, H.; Fan, H.; Wang, Z.; Hu, Y.; Hou, Y. Differential expression of microRNAs in decidua-derived mesenchymal stem cells from patients with pre-eclampsia. J. Biomed. Sci. 2014, 21, 81. [CrossRef]

30. Khatun, R.; Wu, Y.; Kanenishi, K.; Ueno, M.; Tanaka, S.; Hata, T.; Sakamoto, H. Immunohistochemical study of transferrin receptor expression in the placenta of pre-eclamptic pregnancy. Placenta 2003, 24, 870-876. [CrossRef]

31. Yi, T.; Zhou, X.; Sang, K.; Zhou, J.; Ge, L. MicroRNA-1270 modulates papillary thyroid cancer cell development by regulating SCAI. Biomed. Pharmacother. 2019, 109, 2357-2364. [CrossRef]

32. Wei, L.; Li, P.; Zhao, C.; Wang, N.; Wei, N. Upregulation of microRNA-1270 suppressed human glioblastoma cancer cell proliferation migration and tumorigenesis by acting through WT1. OncoTargets Ther. 2019, 12, 4839-4848. [CrossRef]

33. Comes, N.; Buie, L.K.; Borrás, T. Evidence for a role of angiopoietin-like 7 (ANGPTL7) in extracellular matrix formation of the human trabecular meshwork: Implications for glaucoma. Genes Cells 2011, 16, 243-259. [CrossRef]

34. Palmer, K.R.; Tong, S.; Kaitu'u-Lino, T.J. Placental-specific sFLT-1: Role in pre-eclamptic pathophysiology and its translational possibilities for clinical prediction and diagnosis. Mol. Hum. Reprod. 2017, 23, 69-78. [CrossRef]

35. Ackerman, W.; Salomonis, N.; Zhao, G.; Summerfield, T.; Buhimschi, C.S.; Buhimschi, I.A. 477: Preeclampsia (PE) and global dysregulation of placental alternative RNA splicing events. Am. J. Obstet. Gynecol. 2016, 214, S261-S262. [CrossRef]

36. Beavers, K.R.; Nelson, C.E.; Duvall, C.L. MiRNA inhibition in tissue engineering and regenerative medicine. Adv. Drug Deliv. Rev. 2015, 88, 123-137. [CrossRef]

37. Brennan, G.P.; Vitsios, D.M.; Casey, S.; Looney, A.M.; Hallberg, B.; Henshall, D.C.; Boylan, G.B.; Murray, D.M.; Mooney, C. RNA-sequencing analysis of umbilical cord plasma microRNAs from healthy newborns. PLoS ONE 2018, 13, e0207952. [CrossRef]

38. Khoo, C.P.; Roubelakis, M.G.; Schrader, J.B.; Tsaknakis, G.; Konietzny, R.; Kessler, B.; Harris, A.L.; Watt, S.M. miR-193a-3p interaction with HMGB1 downregulates human endothelial cell proliferation and migration. Sci. Rep. 2017, 7, 44137. [CrossRef]

39. Van Craenenbroeck, A.H.; Van Craenenbroeck, E.M. Endothelial progenitor cells and cardiovascular risk: Does ageing trump all other factors? Ann. Transl. Med. 2016, 4, 553. [CrossRef]

40. Carmeliet, P. Angiogenesis in life, disease and medicine. Nature 2005, 438, 932-936. [CrossRef]

41. Carmeliet, P. Angiogenesis in health and disease. Nat. Med. 2003, 9, 653-660. [CrossRef] [PubMed]

42. Kuehbacher, A.; Urbich, C.; Zeiher, A.M.; Dimmeler, S. Role of dicer and drosha for endothelial microRNA expression and angiogenesis. Circ. Res. 2007, 101, 59-68. [CrossRef] [PubMed]

43. Suarez, Y.; Fernandez-Hernando, C.; Pober, J.S.; Sessa, W.C. Dicer dependent microRNAs regulate gene expression and functions in human endothelial cells. Circ. Res. 2007, 100, 1164-1173. [CrossRef] [PubMed]

44. Suarez, Y.; Fernandez-Hernando, C.; Yu, J.; Gerber, S.A.; Harrison, K.D.; Pober, J.S.; Iruela-Arispe, M.L.; Merkenschlager, M.; Sessa, W.C. Dicer-dependent endothelial microRNAs are necessary for postnatal angiogenesis. Proc. Natl. Acad. Sci. USA 2008, 105, 14082-14087. [CrossRef] [PubMed]

45. Tranquilli, A.; Dekker, G.; Magee, L.; Roberts, J.; Sibai, S.; Steyn, W.; Zeeman, G.; Brown, M. The classification, diagnosis and management of the hypertensive disorders of pregnancy: A revised statement from the ISSHP. Pregnancy Hypertens. 2014, 4, 97-104. [CrossRef] [PubMed]

46. Grundmann, M.; Haidar, M.; Placzko, S.; Niendorf, R.; Darashchonak, N.; Hubel, C.A.; von Versen-Höynck, F. Vitamin D improves the angiogenic properties of endothelial progenitor cells. Am. J. Physiol. Cell Physiol. 2012, 303, C954-C962. [CrossRef] [PubMed] 
47. Baker, C.D.; Balasubramaniam, V.; Mourani, P.M.; Sontag, M.K.; Black, C.P.; Ryan, S.L.; Abman, S.H. Cord blood angiogenic progenitor cells are decreased in bronchopulmonary dysplasia. Eur. Respir. J. 2012, 40, 1516-1522. [CrossRef]

48. Agarwal, V.; Bell, G.W.; Nam, J.W.; Bartel, D.P. Predicting effective microRNA target sites in mammalian mRNAs. eLife 2015, 4 . [CrossRef]

49. Schröder-Heurich, B.; von Hardenberg, S.; Brodowski, L.; Kipke, B.; Meyer, N.; Borns, K.; von Kaisenberg, C.S.; Brinkmann, H.; Claus, P.; von Versen-Höynck, F. Vitamin D improves endothelial barrier integrity and counteracts inflammatory effects on endothelial progenitor cells. FASEB J. 2019, 33, 9142-9153. [CrossRef]

50. Liu, K.; Ma, L.; Zhou, F.; Yang, Y.; Hu, H.B.; Wang, L.; Zhong, L. Identification of microRNAs related to myocardial ischemic reperfusion injury. J. Cell. Physiol. 2019, 234, 11380-11390. [CrossRef]

51. Gene Ontology Consortium. Gene ontology consortium: Going forward. Nucleic Acids Res. 2015, 43, D1049-D1056. [CrossRef]

52. Kanehisa, M.; Sato, Y.; Furumichi, M.; Morishima, K.; Tanabe, M. New approach for understanding genome variations in KEGG. Nucleic Acids Res. 2019, 47, D590-D595. [CrossRef]

53. El-Gebali, S.; Mistry, J.; Bateman, A.; Eddy, S.R.; Luciani, A.; Potter, S.C.; Qureshi, M.; Richardson, L.J.; Salazar, G.A.; Smart, A.; et al. The Pfam protein families database in 2019. Nucleic Acids Res. 2019, 47, D427-D432. [CrossRef]

54. Szklarczyk, D.; Gable, A.L.; Lyon, D.; Junge, A.; Wyder, S.; Huerta-Cepas, J.; Simonovic, M.; Doncheva, N.T.; Morris, J.H.; Bork, P.; et al. STRING v11: Protein-protein association networks with increased coverage, supporting functional discovery in genome-wide experimental datasets. Nucleic Acids Res. 2019, 47, D607-D613. [CrossRef] 